infection to return to work, the treatment will be continued until the ova disappear from the fæces.

Bibliography.-Bentley, C. A.: Brit. Med. Jour., 1902, vol. i., p. 190 Boycott and Haldane: Journal of $\mathrm{Hygiene}$, vols. iii. and iv. Boycott : THE LanCET, 1911, vol. i., pp. '18, 783, and 859 . Brumpt, $\mathbf{E}$.: Précis de Parasitologie, Paris, 19i0. Daniels, C. W.: Tropical Medicine and Hygiene, London, 1910 . Dock and Bass : Hookworm Disease, by George Dock, M.D., and Charles C. Bass, M.D., 1910. Looss A.: The Anatomy and Life History of Agchylostoma Duodenale, Dubini Records of the Médicale, 1913, No.7, p. 80. Stockman, R. : Brit. Med. Jour., 1903, vol. ii., p. 189 .

Birmingham.

\section{RADIUM EMANATION THERAPY.}

\section{BY Dr. W. ENGELMANN.}

IN speaking of radium therapy it is best to distinguish between radium therapy in the narrower sense and radium emanation therapy. It is well known that in the former, which may be assumed to be most in vogue in surgery and dermatology, very strong radium preparations are used; the effect is produced by the rays emanating from the radium, the $\alpha, \beta$, and $\gamma$ rays. The effects in question are, therefore, very strong; they resemble those of the $X$ rays of the Roentgen therapy. As examples I may mention the radiating of skin diseases of the most malignant and obstinate type, including even lupus and cancer-in which this treatment is often startlingly successful, and also injections in the case of maliynant tumours, and recently in diseases of the hematopoietic system. In these two examples we have at the same time paradigms for the biological effect of the radium salt on the living tissue, which effect is twofold, namely, irritant-i.e., inflammatory and producing hyperæmia-and, secondly, destructive. In the application of radium emanation, the second kind of radium therapy, called radium emanation therapy, we have a weakened effect. This therapy most interests those who have to deal with internal diseases, and it is of this that $I$ wish to speak in this article.

At Kreuznach, formerly known only for its brine motherlye baths, a certain amount of experience as to the nature of the radium emanation and the manner in which it acts has been acquired during the five years that have elapsed since the systematic practice of radium emanation therapy began; and during these five years the Kreuznach physicians have endeavoured to give themselves and others some account of their experience in an annual collection of essays, the so-called " Radiologische Mitteilungen" ("Radiological Communications"). Not only does Kreuznach possess highly radio-active springs, it is also able to prepare very valuable radium solutions out of the residue obtained in the production of mother-lye; it possesses the only radium factory in Germany.

A number of investigations as to the biological effect of the radium emanation have been made and reported, which do not, indeed, yet give any unobjectionable explanation of the clinical results of the radium emanation therapy, but do justify the hope that such an explanation will one day be found. The nearest approach to a solution of the problem seemed to be made during the investigation of the effects of radium emanation on certain body ferments or on processes in the human or animal organism in which, according to the present teaching of science, enzymotic forces must be supposed to play a part. The actuating influence of radium emanation on the pancreatin, the peptic ferment, and the autolytic ferment is known. The explanation of Van der Velden's observation that radium emanation strikingly increases the coagulability of blood may also be that emanation exercises an actuating influence on certain ferments operative in coagulation.

We may assume a similar influence when we hear that Richet was able to promote the fermentation of lactic acid by a slight dose of emanation and to retard it by a stronger one. The same may be said of the fermentation of yeast, in which an exactly similar, partly retarding, partly promoting effect under the influence of different doses of emanation has been observed.

Von Noorden and Falta believed a connexion to exist between these fermentative processes and what they

1 A lecture given at a clinical meeting of the Municipal Hospitals at Dortmund. observed of the behaviour of the white blood corpuscles under the influence of radium emanation. In the human subject they found after emanation treatment a rise in the number of the white blood corpuscles and then an increased decay. They concluded that, as the white blood corpuscles were supposed to be the seat of the ferments, such a connexion was possible if the actuation of the ferments was really to be regarded as an essential effect of the radium emanation.

These biological influences at any rate show that the relation of radium emanation to the radium preparation as such somewhat resembles that of a concentrated medicament of very violent physiological effect to a diluted one; in the former very strong, violent biological effects, demanding the utmost caution in determining the dose and in the manner of application; in the latter a weakened effect, allowing us to apply the remedy more fearlessly. In certain circumstances the radium rays destroy the tissue, whereas the radium emanation actuates and promotes the processes in the body. In the same sense the increase of the phagocytosis, recently ascertained by Laqueur, is to be valued. Here, too, the question was of therapeutically applied doses. The fact that radium emanation has a retarding effect on the growth of several kinds of bacteria, including pathogenic ones, or even kills them, has been harmoniously ascertained in several different quarters. It cannot be turned to therapeutic account without more ado, because in the test-tube experiment comparatively high doses were given; but it is biologically interesting. Contrariwise Falta and Schwarz have ascertained by experiments in the field of vegetable physiology that even large doses of emanation may have a furthering effect on a physiological process. Emanation has an intensely furthering influence on the growth of oat germs.

It has also been ascertained by Plesch that the maximum pressure of the blood sinks under the influence of radium emanation, and that in some of the cases examined the medium pressure decreased, so that a diminution of the work done by the heart must be assumed. In many persons emanation increases the gaseous interchange, frequently also the elaboration of albumin. Finally, Kikkoji and Bernstein say they have ascertained an increase of the combustion of sugar.

We see, then, that in many cases the effects of radium emanation in experiments and in practical application run parallel. We find that it has an intensely stimulating effect on all the factors of assimilation, that it enhances, as it were, the vital processes. In this sense also I interpreted the results of a series of experiments which I made two years ago. Originally induced by the favourable effects of radium emanation on sufferers from gout, and following von Logham's example, I produced artificial tophi in rabbits. I injected liquid preparations of uric acid into the subcutaneous connective tissue, and observed the behaviour of the deposits under the influence of emanation. Untreated control animals with the same deposits served as objects of comparison. The slow resorption of the deposits which set in in obedience to physiological laws was quite considerably accelerated under the influence of radium emanation, the effect becoming noticeable within 24 hours. At the time when the experiments were made one might have been tempted to regard this phenomenon as a specific effect on gouty deposits, for at that time Gudzent's experiments had not yet been tested. It is known that Gudzent believed himself to have demonstrated that uric acid is found in the blood as urate of mononatrium, and that in two forms-a stable and a less stable one. Under the influence of emanation he found in the test-tube a transformation of the former form into the latter, and believed that in this phenomenon he had discovered an explanation for the removal of the uric acid from the organism, which is really observed. The experiments have been tested in two quarters and not confirmed. This, as is known, was one of the things that led to the rather vehement discussions of last spring, which somewhat discredited the emanation therapy. Unfortunately, or rather, not quite justifiably, for, firstly, Gudzent's experiments were afterwards confirmed. In Madame Curie's laboratory Mesernitzky, who conducted his investigation somewhat differently, found the same results. He worked, it is true, with very high doses of emanation. The question must be declared at least undecided. 
Secondly, however, the good therapeutic results could not be denied. Finally, the fact that the excretion of uric acid was influenced by emanation remained unshaken. Both Gudzent and Mesernitzky were able to ascertain an increase of the excretion of the endogenous uric acid and purin bases in healthy persons. In the case of sufferers from gout it was ascertained that exogenously introduced material containing purin was promptly excreted under emanation treatment, whereas the previous excretion had been slow. This would give us an idea of the manner in which radium emanation operates if we think of the definition of gont, which tells us that gout is the expression of a pronounced disturbance of the fermentative system of the elaboration of purin, which consists in the retardation of the formation, destruction, and secretion of uric acid, and leads to a permanent accumulation of uric acid in the blood, i.e., to uricæmia.

It is to be noted that the persons on whom Gudzent experimented had inhaled the emanation; they were in the so-called emanatories. Mesernitzky found the same results in the laboratory of the salt works belonging to the town of Kreuznach in patients who drank radium water. I must add, it is true, that these phenomena did not appear in all persons with equal intensity, as, indeed, be it said by way of anticipation, in the emanation therapy it is more than ordinarily necessary to individualise. The influencing of the blood is also subject to similar variation. It has been already mentioned that leucocytosis, frequently followed by leucopenia, was ascertained under emanation treatment in von Noorden's clinic. In His's clinic this effect has been ascertained even when the doses were small. My own investigations, it is true, do not confirm this, at least after drink cures. In dogs, on the other hand, when large doses were given, I found, as the Vienna investigators did, quite considerable leucocytosis, which lasted till a fortnight after the last dose. It is true that $I$ gave them doses up to 50,000 units a day (Mache Einheiten); to human beings one gives 1000 to 3000 units (M.E.). In certain forms of diseases those high doses will be more frequently given in future than hitherto. We are reminded in this connexion of the effects of thorium, the sister element of radium, on the hematopoietic system, and of its therapeutic effects in diseases of the blood. It seems to me not impossible that high doses of radium emanation may have a similar effect, but this question has not yet been thoroughly investigated.

A word or two now about the forms of application. We administer emanation by means of baths, inhalation, drinking radium emanation water, rinsing's out, enemas, or compresses. For a while baths did not enjoy among the radium therapeutists the reputation which they decidedly deserved. Quite unintelligibly, if one considers that at watering-places with waters rich in emanation this form of application has long been one of the most favoured. Löwenthal believed that he had ascertained that emanation, which, as is known, has all the characteristics of a gas, does not penetrate through the skin into the body, and that therefore baths are useless. Even if he had been right, his conclusion was a hasty one, for the agent in question was comparatively unknown, not yet exhanstively studied, and, above all, the possibility of the $\alpha, \beta$, and $\gamma$ rays exercising an influence in the form of induced activity, which precipitates itself on the body on all sides, was left out of account. I may remind the reader that emanation, as already said, is a gas which emanates from radium. It is, as is known, colourless, odourless, tasteless, and is best demonstrable in the air, in springs, and in fluids in general by means of the electroscopic method. Emanation electrifies the air, and thus acts, e.g., on the little plates of an electroscope. The measuring method is extremely simple and should always be applied when clinical observations are made after emanation therapeutic measures. The understanding of its behaviour in the organism is facilitated by remembering that emanation is comparatively short-lived. It disappears within about eight days, disintegrating into solid elements, radium $\mathrm{A}, \mathrm{B}, \mathrm{C}, \mathrm{D}$, \&c., which also have a more or less long duration, and emit the biologically effective rays, while they precipitate themselves, if we will conceive it so, on the cells in the organism. Radium emanation water must therefore always be applied as fresh as possible, because otherwise its strength does not correspond to that perhaps ascribed to it.

To return to the baths, I was able three years ago to demonstrate at Strasburger's clinic in Bonn that the emanation does indeed penetrate through the skin during a bath, and is demonstrable in the blood. My measurements were afterwards confirmed from another quarter, and the reputation of baths was restored. According to more recent investigations the penetration of emanation into the body seems to be even more considerable than I then supposed. Thus, also, a new justification was given for the application of poultices, which Strasburger had strongly advocated from the outset. There is, it is true, no doubt that the manner of operation is not thereby exhaustively explained. It is not only the emanation that has penetrated into the body that operates, as does also the inhaled emanation; the $\beta$ and $\gamma$ rays that penetrate through from without operate too. Perhaps also the a rays, which are, indeed, biologically very effective but do not penetrate so deep, operate by irritating the nerve terminations or the like. To be worthy of the name, a radium bath must have a strength of at least 7000 . to 10,000 units (M.E.). Baths containing less than that are not specifically effective. The baths given at Kreuznach contain 22,000 units. If necessary, and if patients can stand it, emanation water can be added till the bath has twice or three times that strength or more. The temperature is that of an ordinary bath, and the patient should stay in it as long as possible. Our patients are not allowed to dry themselves, but rest, wrapped in their bathing-sheets, for 20 to 30 minutes till the emanation dries in, in order to secure the longest possible duration of its influence.

Another method of conveying the radium emanation into the body is by drinking.

After long controversies the opponents of the drinking method now admit that it is at least not inferior in value to inhalation. It is now admitted on all hands that it was, first and foremost, the Kreuznach doctors who always kept emphasising the effectiveness of the drink cures. The opponents contended that when one drinks emanation water it gets after resorption in the intestine only into the small circulation, and that, owing to its instability, the gaseous emanation is so completely exhaled in the lungs that only infinitesimal quantities get into the great circulation. My above-mentioned experiments, in which I gave the animals 60 grammes of emanation water daily -i.e., 600 unitsper os with the cesophageal probang, "spoke strongly, in harmony with the numerous clinical observations that had been made not only at Kreuznach but also elsewhere, for the value of stomachic incorporation. Accurate investigations of the blood-i.e., measurements of the blood with a view to ascertaining how much emanation it contained - which had been made in the most various quarters by Strasburger, Lazarus, and others, proved unambiguously that the drinking method is a very rational one. Not less than two years ago I had ascertained in the case of a dog with an intestinal fistula that even an hour after a dose of emanation water the intestinal juice contained about 30 per cent. of the emanation-content of the dose. It follows that the passing of emanation into the blood lasted for at least that length of time. By measurements of the blood of dogs I was then able to ascertain as a fact that emanation is still demonstrable with certainty in the blood even troo hours after drinking. If, therefore, the patient drinks small quantities of radium emanation water four to six times a day, a lasting loading of the blood with emanation can easily be brought about. And at Kreuznach the method has already stood the test of experience for a long time. In general the patients drink from 1000 to 3000 units (M.E.) a day. of course one must begin with small doses, and increase them only if the water agrees well with the patient.

The third principal form of application is inhalation. The idea of incorporating emanation as gas with the organism by inhalation was obvious. So-called emanatoriums were constructed-little rooms the air of which was loaded with emanation. In these a number of patients conld sit, and thus conveniently inhale emanation. Apparatus was provided for the absorption of bad air and the introduction of oxygen. It was believed that during a patient's stay in such a room the incorporated emanation could be rightly retained in the organism. It is possible that the emanatorium treatment thus came to be overrated, especially when Gudgent claimed to have ascertained that during one's stay in such an emanatorium even an enrichment of the blood with emanation took place. If this were the case, there must be an affinity between emanation and blood. There is, however, as Plesch and I were able to demonstrate by joint experiments, no such affinity. The manner in 
which we experimented even led us to the result that blood takes up about 10 per cent. less emanation than water does. The above-mentioned investigations of the blood by several writers also spoke against the opinion. It is, therefore, probable that an enrichment does not take place, though a plentiful loading of the blood, corresponding to the quantity of emanation used, does. The stay in emanatoriums is, therefore, very well fitted to bring emanation into the body, and therefore to be therapeutically effective. The constructors of emanatoriums have been blamed for bringing only the so-called two-unit emanatoriums into the marketi.e., such as contain two units (M. E.) per litre of air. That is not sufficient, at least for the majority of patients. One must be able to demand from eight to ten units. Von Noorden complains that they do not adequately meet the necessity of adapting the doses to the requirements of the individual patients. He prophesies a greater future for the inhaler designed for only one person. It is portable, and can be used by patients who are confined to bed ; it is also cheaper, allows one to vary the dose, and does not compel patients to sit together in a little room in which the air is often bad.

Along with Professor Bickel I some time ago perfected, tested, and showed to the Medical Society of Berlin such an inhaler intended for one person. It is intended to serve as supplementary to any apparatus for the preparation of emanation water-Neumann's activator, for instance. In my opinion one is best able to practise rational radinm emanation therapy if one uses two such apparatuses, no matter of what system. One has the option of giving radium baths, poultices with radium water, rinsings out, enemas, nutrient enemas, emanation water to be drunk, and, lastly, emanation to be inhaled with the help of an inhaler intended for the use of one person.

We come now to the groups of diseases for which radinm emanation therapy is suited, and here the first disease to be named is gout, genuine gout, arthritis urica, for which radium emanation seems in many cases to be almost a specific remedy.

I remind the reader of the above remarks on the influencing of the assimilation of purin. The best treatment is that in which all the above-mentioned forms of application-drinking, inhalation, baths, compresses if necessary-are cantiously combined. If the organism reacts-it is a singular fact that there are not a few cases in which it does not-it frequently happens that the feeling that it is taking effect soon sets in. After from four to six days the known reactions take place, pains in the joints in the most different parts of the body, often as severe as in a typical attack of gout, but for the most part of shorter duration. Antipyretics and atophan may be given for a time. Then the reaction abates, and the effect slowly sets in. Subjective and objective improvement takes place. In contradiction to von Noorden I wish to state that the tophi do become softer. Strasburger and others have also noticed this. The treatment-in cases of necessity a drink cure alone suffices-should last six weeks, and it seems advisable to repeat a drink or inhalation cure once or twice a year, the choice being determined by the remedial resources of the patient's place of residence.

Another large field for the advantageous practice of emanation therapy is articular rheumatism in its most various forms, above all the primarily chronic polyarthritis exsudativa and the primarily chronic arthritis sicca, which occurs, I presume, mostly at an advanced age. The treatment is the same as that sketched above, but here the use of baths and poultices is very decidedly to be recommended. Under certain circumstances the abvisability of injecting radium solutions is also to be considered. Here, too, there may be slight reactions-pains and sometimes a slight increase of temperature. Sciatica, too, is a malady in which the treatment is likely to be rewarded by success, but owing to the multiformity of its etiology there are, of course, unsuitable cases. When all other therapeutic measures fail a trial is much to be recommmended. I have seen cures and many partial cures myself. In general the effects on many other disorders of the nervous system are notable. This fact is probably connected, as Strasburger points our, with the antiphlogistic and resorption-furthering qualities of emanation. Moreover von Knaffel-Lenz, of Vienna. professes to have ascertained by experiment that the healthy nerve tissue is influenced by emanation. All kinds of neuritis and neuralgiu are suitable objects of treatment. Here, too, besides drink and inhalation cures, baths and compresses play a great part. The effect of the latter, e.g., is often subjectively ascertainable immediately after application, often diagreeably at first. I have seen two extremely obstinate cases of herpes zoster in persons fairly advanced in years, which had defied every kind of treatment, quite surprisingly bettered. These are only instances among many. The lancinating pains of sufferers from tabes dorsalis are relieved. Then there is the myalgia group. Besides all these, I mention also bronchial asthma, presclerosis, arterio-sclerosis, and diseases of the genitals of women, especially in cases in which it is important to assist the resorption of chronic exudates, swellings of tissue, and the like. And, in general, adhesions, tissue thickenings of every kind, formation of callus, formations of scars cannot be otherwise than favourably influenced by a combined local and general emanation treatment. Advantageous results after radiation with radium preparations in cases of myoma and disturbance in menstrua tion have quite recently been reported from the Freiburg clinic for women. In these cases the radiation with radium rays took the place of radiation with Roentgen rays. It seems to me not impossible that the somewhat milder application of these rays practised in radium emanation therapy will in future play a more important part in the treatment of women. The experience gained at Kreuznach, where female patients are very numerous, speaks strongly for this opinion. But the decisive, quite objective observations cannot be made at a watering-place. At any rate, a histologically demonstrable influencing of the ovaries and also the occurrence of miscarriage after mere drinking of emanation water have already been experimentally ascertained. By means of poultices, rinsings out, and, above all, nutrient enemas, the use of which is already very frequent at Kreuznach, it will also be possible to render the general radium treatment of the diseases of women very powerful local support.

The skin diseases belong in general to the province of radium therapy in the narrower sense--i.e., they must be treated by radiation with radium preparations. In many obstinate cases, however, radium emanation also may well deserve to be recommended. I will not enumerate all the maladies for which the emanation treatment is suitable, but only mention some cases of cured or very considerably bettered scleroderma reported in the literature of the subject. One such case $I$ saw myself. So far as I am aware such cases defy every other treatment.

I do not mean to say that the treatment in question is, as a matter of course, the only right treatment for all the phenomena of disease which $I$ have somewhat copiously enumerated, but only that in suitable cases it is reasonable and worth while to try it. After all, this therapy is still young, and it is possible that our views as to the question what diseases it is suitable for may undergo considerable modification. Von Noorden thinks it is applicable to still more diseases than I have mentioned. He believes, for example, that attempts to cure corpulence on the lines of the above-mentioned experiments concerning the enhancement of fundamental assimilation would have a great chance of success. Of the treatment of certain diseases of the stomach he thinks the same; he has seen good results in cases of nervous gastritis. A fellow doctor drew my attention to the fact that his achylia gastrica, which had greatly impaired his health and defied all treatment, had so considerably improved after a drink cure that he now, months afterwards, felt better than ever. I believe that strictly clinically conducted experiments in this direction will not fail to be rewarded.

Finally, I mention the effect of emanation on the power of generation. The observations come from the most various quarters, above all from von Noorden and from doctors at Joachimsthal and Kreuznach. The phenomenon would harmonise very well with the results of $O$. Hertwig's investigations, who ascertained a quite specific effect of the radium rays on the nuclear substances of the two sexual cells, and with the observations of Halben, who succeeded in enhancing the secondary symptoms of sexual passion in water-newts by radium emanation. Affection of the kidneys, tendency to bleeding, and tendency to miscarriage are regarded for the present as contra-indications.

I conclude my statements and enumerations, which, owing to the shortness of the time at my disposal, cannot be 
complete, and which aim only at giving a general idea of the nature of radium emanation therapy. I believe one need not be too much of an optimist to be able to endorse von Noorden's declaration that radium emanation therapy may now be justly regarded as belonging to the solid stock of medical therapeutic measures.

Bad Kreuznach.

\section{THE USE OF TUBERCULIN IN DIAGNOSIS AND TREATMENT. ${ }^{1}$}

\section{By J. F. HALLS Dally, M.A., M.D. Cantab.,} M.R.C.P. LOND.,

PHYSICIAN, AND PHYSICTAN IN CHARGE OF THE SPECIAL DEPARTMENT FOR TUBERCULOSIS, TO THE ST. MARYLEBONE GENERAL DISPENSARY; SENIOR ASSISTANT PHYSICIAN TO THE NATIONAL HOSPITAL FOR DISEASES OF 'THE HEAR'T.

Tuberoulin in diagnosis. - The specific tuberculin tests are four in number : (1) the ocular reaction of Wolff-Eisner and Calmette; (2) the cutaneous reaction of von Pirquet; (3) the percutaneous reaction of Moro; and (4) the subcutaneous reaction of Koch. All the above tests have their appropriate individual uses. They depend on the existence of that " paradox reaction" termed anaphylaxis.

1. Calmette's ocular reaction, described in 1307 , has since then attracted considerable attention. Many precautions are necessary in its application, and, even so, the test is not without a certain measure of risk to the eye, so that both for this reason and because, moreover, it is not as sensitive as the other three tests I have mentioned, it has now largely fallen into disuse in general medicine.

2. Von Pirquet's cutaneous reaction. The chief value of this reaction appears to be in the case of children under 5 years of age, where a positive reaction may be taken to indicate the presence of definite tuberculosis. In adolescents and adults a positive result must be reviewed in conjunction with all other available data before an opinion be given.

3. Moro's percutaneous reaction is a modified von Pirquet's reaction, and is readily performed by rubbing into a small portion of the skin an ointment of equal parts of old tuberculin and anhydrous lanolin. Occurrence of a localised dermatitis denotes a positive reaction. The ease and safety of this test are its chief advantages.

4. Koch's subcutaneous test is necessarily preceded by the patient's temperature being recorded three-hourly for at least three days before the injection, since this test should only be applied if the temperature is below $99^{\circ} \mathrm{F}$. For adults the first test dose consists of 0.001 c.c. old tuberculin (O.T). If no reaction ensues 0.005 c.c. O.T. is given in three days, and if still negative 0.01 c.c. O.T. at a like interval of time. In the continued absence of reactions one may say that no tuberculosis is present. For children over about 10 years of age the diagnostic dose may often be equivalent to that for adults, but for a child of 5 years of age the initial dose should not exceed 0.0005 c.c. O.T. and the next subsequent dose 0.0025 c.c. O.T.

The complete and typical reaction which may be expected, if tuberculosis is present, is in nature tripartite-viz., focal, local, and general, these manifestations being given in the order of importance. The focal reaction is essentially specific and produces hyperæmia and inflammatory swelling around the tuberculous focus, this in the lung being evidenced by the presence of new or increased moist crepitations in the affected patch, and appearance or increase of tubercle bacilli and elastic fibres in the sputum, which may be, and often is, itself increased in quantity. I regard the focal reaction as by far the most definite and conclusive of the three. Next comes the local reaction at the point of injection, which gives similar clinical appearances (apart from the papula) to the von Pirquet reaction. The general reaction is shown by fever and consequent malaise, sc. A rise of $10 \mathrm{~F}$. or more may be regarded as positive, and this comes on about 8 to 12 hours after the injection, appearing usually on the temperature chart as a peak with sharp elevation and decline, the downward slope at times being interrupted by a second rise

1 Abstract of a paper read at a mecting of the Chelsea Clinical Society on April 15th, 1913 . usually of lower, but occasionally of higher, level than the first.

Of all the diagnostic tests the subcutaneous one is, in my experience, the most reliable. If no tuberculosis is present tuberculin has no ill-effects. This fact alone encourages one to use tuberculin at the earliest moment to pick out cases suitable for sanatorium and tuberculin treatment from those for which these remedial methods are either inadvisable or unnecessary.

Tuberculin in treatment. - In the brief space at my disposal I cannot hope to do more than touch upon a few salient points based upon clinical observation in my tuberculosis department. The preparations of tuberculin are many and various. I find that some patients are more sensitive to one form of tuberculin and less sensitive to another, so that if intolerance of one kind of tuberculin is manifested it is wise to make a change. The main point to remember is that, no matter what preparation is used, sufficient time must be allowed for proper spacing of doses, and that it is never good policy to attempt to proceed too rapidly. If the remedy is to be beneficial, I believe that throughout one should try to avoid all but slight reactions, the only exception being in early stages of the weak preparations, where hypersensitiveness can be overcome by cautious increase of dosage at comparatively short intervals. This has the effect of inducing tolerance to the successive stronger doses, whereas repetition of the same or smaller doses at longer intervals appears to produce, and, if present, to intensify, that very condition of extreme sensitiveness which one is so anxious to avoid. Hence under such conditions one's motto must be, "Vestigia nulla retrorsum." Should, however, such procedure fail, the only course is to suspend the treatment for some weeks.

As to the scale of dosage which should be adopted there is no hard-and-fast rule. It is impossible briefly to give a set scale to which one should work, for not only has one to deal with each individual patient as a separate problem, bot also to be guided by the ever-varying factors of the patient's sensitiveness in particular and of his state in general. In my own practice I have abandoned other methods for the intensive system of dosage. In the treatment of pulmonary tuberculosis I begin with small doses of a weak bovine preparation-e.g., 1/10000 c.c. or less of P.T.O. - and working through increasing doses of this to bovine old tuberculin (P.T.). I complete the treatment in all cases where necessary by a course of human tuberculin, usually 0. T. or albumose-free old tuberculin (T.A.F.), finishing with doses of 1 c.c. or even more at longer intervals. In the earlier stages of treatment my custom is to inject every three or four days, and when larger doses of the strong preparations are reached, once a week.

Since the administrator cannot, with any accuracy, check the number of milligrammes of solid active substance con tained in the particular preparation used, I invariably record dosage in terms of cubic centimetres, for one can always measure exactly the amount of fluid that one injects. Tuberculin, when diluted, soon becomes inert, so that fresh dilutions should be made at least once a week. I nearly always make the dilution with freshly prepared saline solution containing 0.5 per cent. phenol at the time of administration. This procedure takes a little longer, but one thus avoids severe reactions consequent upon a potent dose following one which has been inactive.

On these lines immunisation can usually be attained within from 4 to 12 months, and various statistics show that such immunisation is capable of persisting over a period of several years.

In cases which are doing well the dosage can be increased more rapidly, so that the total time of immunisation is thus shortened. In advanced cases tuberculin does good at times in checking the rapid onward march of the disease, and the temperature may be brought to normal with apparent amelioration of physical signs to percussion and anseultation. Other cases, again, do badly. X ray examination in both these classes not infrequently reveals increase in shadow in the lungs, but in a case doing well in other respects it would be hard to say that such increased opacity represents extension of disease, since augmentation of visible shadow may quite well be due to attempt at repair by fibrosis. Should the disease progress notwithstanding cautious injections, tuberculin should be discontinued.

Lower Seymour street, W. 